\title{
Perfil de permeabilidade em concretos refratários
}

\section{(Permeability gradient in refractory castables)}

\author{
M. D. M. Innocentini, A. R. Studart, R. G. Pileggi, V. C. Pandolfelli \\ Departamento de Engenharia de Materiais \\ Universidade Federal de S. Carlos - UFSCar \\ Rod. Washington Luiz, km 235, S. Carlos, SP, 13565-905 \\ pmmi@iris.ufscar.brouvicpando@power.ufscar.br
}

\begin{abstract}
Resumo
A durabilidade de um concreto refratário está diretamente associada à facilidade com que gases e líquidos corrosivos penetram em sua estrutura porosa. Muitas vezes, contudo, a resistência ao escoamento não é uniforme no interior do material, podendo levar a resultados não representativos do corpo como um todo, dependendo da região de análise. Neste trabalho, o perfil de permeabilidade em função da profundidade foi investigado para concretos refratários autoescoantes com ultrabaixo teor de cimento obtidos em diferentes temperaturas de tratamento térmico. Os resultados permitiram concluir que a eliminação de água de hidratação, a sinterização e o destacamento da matriz-agregado são os mecanismos principais responsáveis pela variação da permeabilidade entre $110^{\circ} \mathrm{C}$ e $1650^{\circ} \mathrm{C}$. A leve sedimentação dos agregados para o interior da amostra durante a moldagem e o empacotamento diferenciado da matriz na superfície exposta à atmosfera explicam o gradiente de permeabilidade observado nos concretos.
\end{abstract}

Palavras-chave: permeabilidade, concreto refratário, sinterização, microtrincamento, secagem.

\section{INTRODUÇÃO}

Concretos refratários são geralmente conformados em uma variedade de geometrias e tamanhos, tornando difícil a medição inloco de suas propriedades físicas. Ensaios em laboratório com pequenas amostras são assim usados para obter informações sobre o material que são então extrapoladas para situações reais na indústria. A principal hipótese neste caso é que todas as propriedades nas amostras (geralmente cilíndricas ou cúbicas), tais como porosidade, resistência mecânica e permeabilidade, são representativas e comparáveis às de peças de grande porte e de formato complexo.

Dentro deste contexto, uma questão pouco investigada na literatura é se a permeabilidade varia da superfície para o interior do corpo, devido principalmente ao modo como matriz e agregado acomodam-se durante o empacotamento. Tal informação é especialmente útil no caso em que há segregação das matérias-primas

\begin{abstract}
The durability of refractory castables is ultimately related with the ability of fluids to penetrate and interact with the porous structure. In some cases, however, the flow resistance is not uniform within the refractory body, and a global analysis may result in misleading information about the refractory properties depending on the size and position of the sample. In this work, the permeability gradient was measured along the thickness of a self-flow ultra-low cement refractory castable as a function of the thermal treatment temperature. Results led to the conclusion that dehydration, sintering and microcracking are the key mechanisms for the variation in the permeability. The minor sedimentation of aggregates during casting and the better matrix packing on the sample surface explain the lower permeability at the surface exposed to atmosphere during the castable molding.
\end{abstract}

Keywords: permeability, refractory castables, sintering, microcracks, dehydration.

cerâmicas durante a conformação da peça. Neste caso, o excesso de partículas finas (matriz) na superfície da amostra pode gerar uma camada de menor permeabilidade que a do interior, onde há maior quantidade de agregados. Uma vez que as propriedades medidas são geralmente assumidas uniformes ao longo da espessura do corpo, com a desconsideração da presença de tal camada superficial pode-se superestimar a resistência ao escoamento de fluidos no interior do corpo cerâmico. A presença da camada de baixa permeabilidade durante a secagem pode também causar o aumento da pressão do vapor, danificando a estrutura interna do refratário [1-5]. Em uso, o desgaste da camada superficial por abrasão ou corrosão pode revelar ainda uma camada interior pouco protegida contra o ataque de escórias [1, 2].

Especificamente neste trabalho, o objetivo é verificar se um concreto refratário auto-escoante de ultrabaixo teor de cimento apresenta permeabilidades distintas em sua superfície e em seu interior e como as mesmas são afetadas pela temperatura de tratamento térmico. 


\section{MATERIAIS E MÉTODOS DE ANÁLISE}

Duas amostras de concreto refratário auto-escoante com dimensões de 7,5 cm de diâmetro e cerca de $15 \mathrm{~cm}$ de altura e composição consistindo de uma fina matriz $(0,1 \mathrm{a} 100 \mu \mathrm{m})$ e agregados (100 $\mu \mathrm{m}$ a $4,7 \mathrm{~mm})$, com alto teor de alumina (>99\%) e ultrabaixo teor de cimento $(0,3 \% \mathrm{CaO})$ foram preparadas com distribuição granulométrica seguindo o modelo de Andreasen $(q=0,21)$. A moldagem foi realizada verticalmente aplicando-se pequena vibração em moldes cilíndricos de PVC apoiados em uma base metálica, permanecendo apenas a superfície superior do concreto exposta à atmosfera. Após cura por 24 horas a $40^{\circ} \mathrm{C} \mathrm{e} 98 \%$ de umidade, os corpos-de-prova foram secos em estufa por 24 horas a $110^{\circ} \mathrm{C}$. Uma das amostras foi então tratada termicamente a $1200{ }^{\circ} \mathrm{C}$ com tempo de patamar de 24 horas. Foram utilizadas taxas de aquecimento lentas $\left(1^{\circ} \mathrm{C} / \mathrm{min}\right.$ até $600{ }^{\circ} \mathrm{C}, 2^{\circ} \mathrm{C} / \mathrm{min}$ até $900{ }^{\circ} \mathrm{C}$ e $3{ }^{\circ} \mathrm{C} / \mathrm{min}$ até a temperatura de queima) para evitar quaisquer danos aos corpos durante o tratamento térmico [3]. Ambas amostras foram então fatiadas radialmente com auxilio de um disco diamantado, fornecendo 5 corpos com espessura de cerca de 2,5 cm. A fatia inferior de cada amostra (apoiada na base metálica) foi desprezada para efeito de análise por ter apresentado trincas durante o processo de corte. A Fig. 1 apresenta esquematização do corte de cada amostra cilíndrica e o posicionamento de cada fatia.

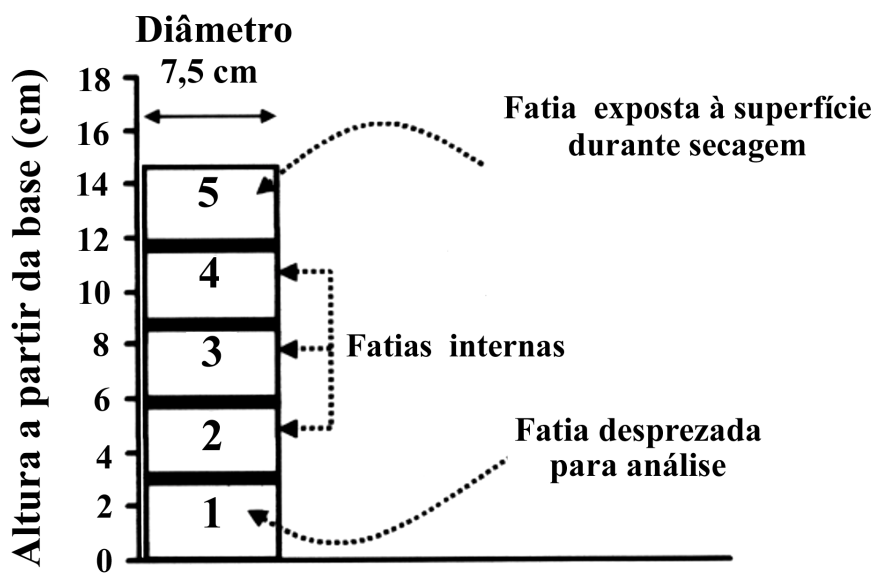

Figura 1: Detalhes do corte de cada amostra em 5 fatias para análise de permeabilidade.

[Figure 1: Scheme of sample slices for permeability analysis].

Todas as 4 fatias restantes de cada amostra (1 superficial e 3 internas) tiveram sua permeabilidade ao ar determinada experimentalmente em equipamento esquematizado na Fig. 2 conforme método detalhado [2, 6]. O sentido do escoamento foi o mesmo em todas as fatias (ascendente). Os dados experimentais foram ajustados segundo a equação de Forchheimer para fluidos compressíveis [2, 6-9]:

$$
\frac{\mathrm{Pe}^{2}-\mathrm{Ps}^{2}}{2 \mathrm{P}_{\mathrm{s}} \mathrm{L}}=\frac{\mu \mathrm{v}_{\mathrm{s}}}{\mathrm{k}_{1}}+\frac{\rho \mathrm{v}_{\mathrm{s}}^{2}}{\mathrm{k}_{2}}
$$

na qual $\mathrm{P}_{\mathrm{e}}$ e $\mathrm{P}_{\mathrm{s}}$ são respectivamente as pressões absolutas medidas na entrada e na saída da amostra, $\rho$ e $\mu$ são respectivamente a densidade e a viscosidade do ar, $\mathrm{v}_{\mathrm{s}}$ é a velocidade superficial do ar, calculada dividindo-se a vazão volumétrica $Q$ pela área frontal e livre para escoamento A $\left(2,54 \times 10^{-3} \mathrm{~m}^{2}\right)$ e $\mathrm{k}_{1} \mathrm{e}_{2}$ são constantes de permeabilidade, conhecidas respectivamente como permeabilidades Darciana e não-Darciana.

As constantes de permeabilidade, propriedades dependentes apenas do meio poroso, foram obtidas neste trabalho por ajuste dos valores experimentais de $\mathrm{v}_{\mathrm{s}}$ versus $\left[\mathrm{P}_{\mathrm{e}}{ }^{2}-\mathrm{P}_{\mathrm{s}}{ }^{2}\right] / 2 \mathrm{P}_{\mathrm{s}} \mathrm{L}$ na equação (A) através do método dos mínimos quadrados. Considerou-se $\mu_{\mathrm{ar}}=1,8 \times 10^{-5} \mathrm{~Pa} . \mathrm{s}$ e $\rho_{\mathrm{ar}}=1,08 \mathrm{~kg} / \mathrm{m}^{3}$ para $\mathrm{T}_{\mathrm{amb}}=25^{\circ} \mathrm{C}$ e $\mathrm{P}_{\mathrm{atm}}=\mathrm{P}_{\mathrm{s}}=690 \mathrm{mmHg}$. Todas as medidas de vazão e pressão em cada ensaio foram realizadas em tréplica para verificar a reprodutibilidade dos valores. Cada amostra foi analisada uma vez, fornecendo apenas uma curva de permeabilidade.

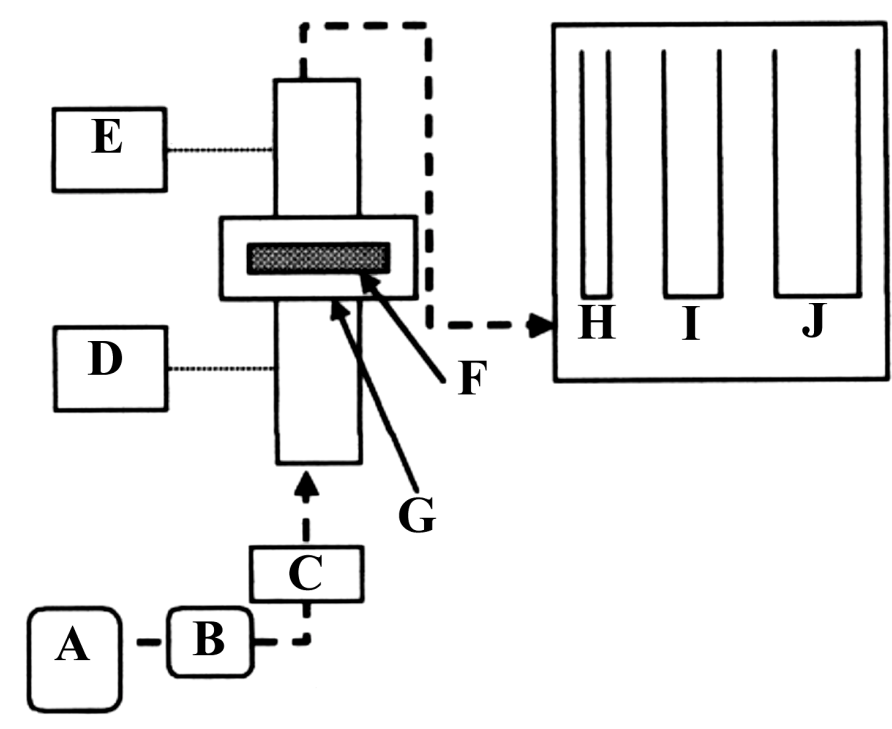

A - Compressor de ar, $2 \mathrm{Hp}$

B - Filtro para óleo e umidade

C - Válvula de controle de pressão

$\mathrm{D}$ - Transdutor e leitor de pressão, $\mathrm{P}=0$ a $10 \mathrm{bar}$

E - Manômetro, $\Delta \mathrm{P}=0$ a $500 \mathrm{mmH}_{2} \mathrm{O}$

$\mathrm{F}$ - Amostra cilíndrica $(\mathrm{d}=7.5 \mathrm{~cm}, \mathrm{~L}=2.5 \mathrm{~cm})$

G - Porta-amostras (diâmetro útil $=5.7 \mathrm{~cm}$ )

$\mathrm{H}$ - Bolhômetro, $\mathrm{Q}=0.7$ a $36 \mathrm{~mL} / \mathrm{min}$

I - Bolhômetro, $\mathrm{Q}=60$ a $600 \mathrm{~mL} / \mathrm{min}$

$\mathrm{J}$ - Bolhômetro, $\mathrm{Q}=0.5$ a $14 \mathrm{~L} / \mathrm{min}$

Figura 2: Detalhes esquemáticos do aparato usado neste trabalho para medição da permeabilidade de concretos refratários.

[Figure 2: Schematic details of permeameter used in this work].

Após os ensaios de permeabilidade, as fatias resultantes do corpo tratado a $110{ }^{\circ} \mathrm{C}$ foram calcinadas a $900{ }^{\circ} \mathrm{C}$ e aquelas previamente queimadas a $1200^{\circ} \mathrm{C}$ foram tratadas termicamente a $1650^{\circ} \mathrm{C}$. Foram mantidas as mesmas taxas de aquecimento e patamares de queima anteriormente usados.

Novos ensaios de permeabilidade foram então realizados para verificar as alterações resultantes da queima dos corpos em nova temperatura. A Fig. 3 ilustra o procedimento adotado para a preparação e análise das amostras neste trabalho. 


\section{RESULTADOS E DISCUSSÃO}

A Fig. 4 apresenta os valores das constantes de permeabilidade obtidos para cada uma das fatias das amostras tratadas a $110^{\circ} \mathrm{Ce} 900^{\circ} \mathrm{C}$.
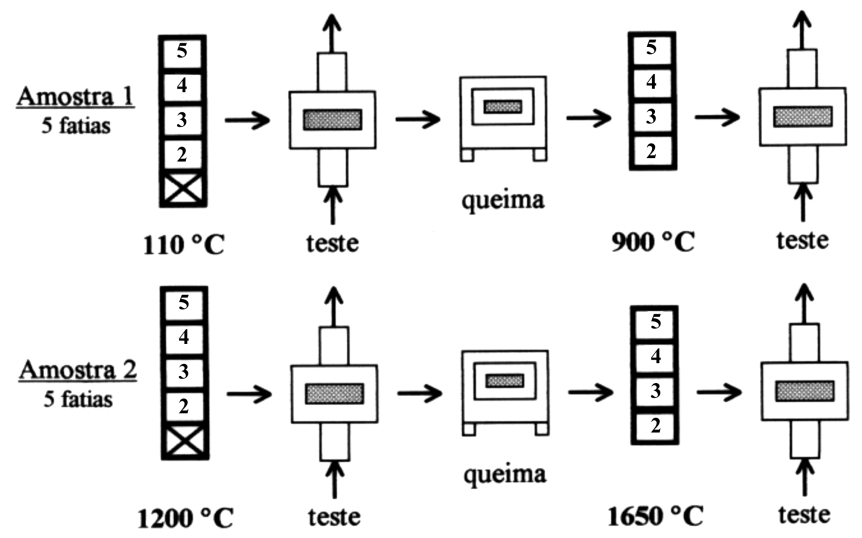

Figura 3: Esquema de preparação e análise das amostras neste trabalho.

[Figure 3: Schematic procedure for sample preparation and analysis used in this work].

Na Fig. 4-a observa-se a evolução de $\mathrm{k}_{1}$ ao longo da posição da fatia quando a amostra inicialmente seca a $110{ }^{\circ} \mathrm{C}$ foi queimada a $900^{\circ} \mathrm{C}$. Nota-se que a $110^{\circ} \mathrm{C}$, a fatia superior, exposta à atmosfera durante a secagem, teve a permeabilidade claramente menor do que as das fatias internas da amostra $\left(\mathrm{k}_{1,110^{\circ} \mathrm{C} \text {,sup }}=0,67 \times 10^{-16} \mathrm{~m}^{2}\right)$. Estas, por sua vez, apresentaram nível de permeabilidade praticamente invariável ao longo da espessura do corpo $\left(\mathrm{k}_{1,110^{\circ} \mathrm{C} \text {,int }}=0,95 \pm\right.$ $0,04 \times 10^{-16} \mathrm{~m}^{2}$ ). Após queima a $900{ }^{\circ} \mathrm{C}$, a diferença entre valores na superfície $\left(\mathrm{k}_{1,900^{\circ} \mathrm{C} \text {,sup }}=4,28 \times 10^{-16} \mathrm{~m}^{2}\right)$ e no interior $\left(\mathrm{k}_{1,900^{\circ} \mathrm{C} \text {,int }}=4,50 \pm 0,29 \times 10^{-16} \mathrm{~m}^{2}\right)$ diminuiu, embora a permeabilidade tenha aumentado em valores absolutos em relação às amostras a $110^{\circ} \mathrm{C}$.

A Fig. 4-b apresenta tendências similares para a variação da constante de permeabilidade não-Darciana, $\mathrm{k}_{2}$, em relação à posição da fatia e à temperatura de queima. $\mathrm{A} 110^{\circ} \mathrm{C}$, o valor médio de $\mathrm{k}_{2}$ foi $220 \%$ superior no interior do corpo-de-prova do que em sua fatia superficial. Tal diferença caiu drasticamente para apenas 3,1\% quando as amostras foram queimadas novamente na temperatura de $900{ }^{\circ} \mathrm{C}$. O desvio padrão de $\mathrm{k}_{2}$ entre as 3 fatias internas do corpo passou de $18 \%$ a $110^{\circ} \mathrm{C}$ para cerca de $22 \%$ a $900{ }^{\circ} \mathrm{C}$.

Comparativamente, o valor médio de $\mathrm{k}_{1}$ aumentou 5 vezes de $110^{\circ} \mathrm{C}$ para $900{ }^{\circ} \mathrm{C}$ enquanto $\mathrm{k}_{2}$ teve um aumento mais significativo (cerca de 30 vezes). A explicação para esse comportamento está relacionada à eliminação de água das fases hidratáveis contidas no concreto com a elevação de temperatura de queima [3]. Uma vez que entre $110^{\circ} \mathrm{C}$ e $900^{\circ} \mathrm{C}$ o processo de sinterização ainda não se iniciou para a composição do concreto analisada neste trabalho, todos os vazios deixados pelas moléculas de água permaneceram inalterados e passaram a ser um trajeto adicional para o escoamento do ar. A ocorrência deste comportamento pode ser reforçada comparando-se a variação diferenciada entre as constantes de permeabilidade. Enquanto $\mathrm{k}_{1}$ no termo linear $\left(\mu \mathrm{v}_{\mathrm{s}} / \mathrm{k}_{1}\right)$ da equação de Forchheimer reflete a influência da área de interação sólido-fluido, $\mathrm{k}_{2}$ pode ser relacionado no termo parabólico $\left(\rho \mathrm{v}_{\mathrm{s}}^{2} / \mathrm{k}_{2}\right)$ com o efeito da tortuosidade do meio poroso e com a turbulência gerada pelo mesmo
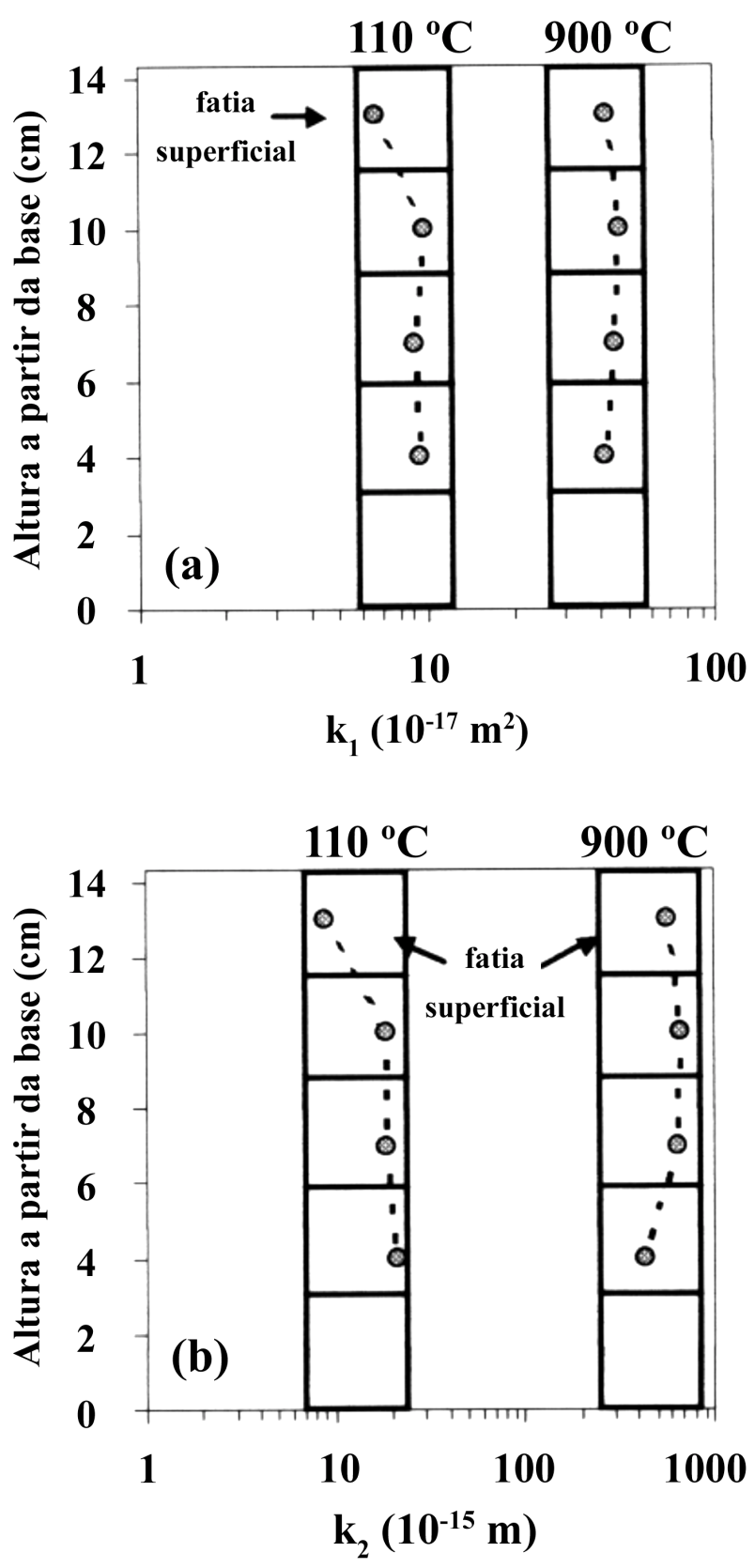

Figura 4: Variação das constantes de permeabilidade ao longo da espessura do concreto refratário tratado a $110^{\circ} \mathrm{C}$ e $900^{\circ} \mathrm{C}$. (a) Permeabilidade Darciana, $\mathrm{k}_{1}$. (b) Permeabilidade não-Darciana, $\mathrm{k}_{2}$.

[Figure 4: Permeability profile along the castable thickness for sample treated at $110^{\circ} \mathrm{C}$ and $900^{\circ} \mathrm{C}$. (a) Darcian permeability, $k_{1}$. (b) Non-Darcian permeability, $\left.k_{2}\right]$.

sobre o fluido [6]. Assim, o aumento predominante observado em $\mathrm{k}_{2}$ no interior da amostra com o aumento de temperatura de $110^{\circ} \mathrm{C}$ para $900{ }^{\circ} \mathrm{C}\left(\mathrm{de}_{2} / \mathrm{k}_{1,110^{\circ} \mathrm{C}}=20,6 \mathrm{~m}^{-1}\right.$ para $\left.\mathrm{k}_{2} / \mathrm{k}_{1,900^{\circ} \mathrm{C}}=130,5 \mathrm{~m}^{-1}\right)$ confirma a formação de um caminho para o fluido predominantemente menos tortuoso com a saída da água de hidratação.

Em relação à diferença de valores das constantes $\mathrm{k}_{1}$ e $\mathrm{k}_{2}$ da fatia superficial para aquelas no interior do corpo-de-prova, a explicação está correlacionada ao processo de acomodação das partículas durante a conformação do concreto. Durante essa etapa é possível que ocorra uma leve sedimentação de agregados para o interior da amostra, dependendo principalmente do teor de água adicionado 
durante a mistura e do volume de matriz do sistema, deixando uma camada predominante de finos (matriz) na superfície. Por apresentar uma mobilidade maior, as partículas desta camada podem ter uma melhor acomodação (alinhamento) de modo a minimizar a energia superficial, diminuindo a área de interface ar-partícula. Além disso, a ausência de partículas grosseiras na camada superficial impede a formação de poros interconectados nesta região, como aqueles formados no interior do corpo ao longo da interface matriz-agregado [10]. Todos esses fatores contribuem para geração de uma camada com fração reduzida de poros de diâmetro inferior aos poros interiores da estrutura e conseqüentemente com menor permeabilidade. É importante ressaltar que a sedimentação dos agregados mencionada restringe-se apenas à região superficial das amostras e não se caracteriza como um fenômeno de segregação, que não foi observado ao longo dos corpos produzidos.

$\mathrm{O}$ aumento da permeabilidade superficial com o aumento da temperatura de $110^{\circ} \mathrm{C}$ para $900^{\circ} \mathrm{C}$ pode ser explicado pela eliminação da água de hidratação. Neste caso, a variação encontrada entre as constantes na superfície $\left(\mathrm{de}_{2} / \mathrm{k}_{1,110^{\circ} \mathrm{C}}=13,4 \mathrm{~m}^{-1}\right.$ para $\mathrm{k}_{2} /$ $\mathrm{k}_{1,900^{\circ} \mathrm{C}}=133,1 \mathrm{~m}^{-1}$ ) foi bem próxima àquela verificada para as fatias internas, confirmando que a saída de água é um dos mecanismos de geração de poros permeáveis.

A Fig. 5 apresenta resultados da análise de permeabilidade para as amostras tratadas a $1200{ }^{\circ} \mathrm{Ce} 1650^{\circ} \mathrm{C}$. Nota-se na Fig. 5-a tendência similar àquela verificada para a permeabilidade Darciana nas amostras tratadas a $110^{\circ} \mathrm{C}$ e $900{ }^{\circ} \mathrm{C}$. A $1200{ }^{\circ} \mathrm{C}$, a fatia superficial apresentou valor de $\mathrm{k}_{1} 51 \%$ mais baixo do que o valor médio das fatias internas. Tal redução praticamente se manteve (48\%) com a queima das amostras a $1650{ }^{\circ} \mathrm{C}$. Internamente, o valor de $\mathrm{k}_{1}$ para as 3 fatias manteve-se praticamente constante $\left(47,6 \pm 0,5 \times 10^{-16} \mathrm{~m}^{2}\right)$ na amostra de $1200^{\circ} \mathrm{C}$ e também na amostra queimada a $1650{ }^{\circ} \mathrm{C}$ $\left(134,2 \pm 9,8 \times 10^{-16} \mathrm{~m}^{2}\right)$, indicando que todo o fenômeno de redução de permeabilidade ocorre na camada superficial dos concretos.

Na Fig. 5-b, novamente é possível observar tendência clara em relação à variação da permeabilidade não-Darciana da superfície para o interior das amostras. $\mathrm{Na}$ amostra queimada a $1200^{\circ} \mathrm{C}, \mathrm{k}_{2}$ na superfície corresponde à apenas $15 \%$ do valor médio encontrado nas fatias internas, enquanto a $1650{ }^{\circ} \mathrm{C}$ esse valor passa a $22 \%$. $\mathrm{O}$ desvio padrão de $\mathrm{k}_{2}$ entre as fatias internas também foi pequeno $\left(10 \%\right.$ a $1200{ }^{\circ} \mathrm{C} \mathrm{e} 12 \%$ a $\left.1650{ }^{\circ} \mathrm{C}\right)$, permitindo considerar essa propriedade constante no interior das amostras.

Em valores absolutos, a permeabilidade $\mathrm{k}_{2}$ média no interior do corpo queimado a $1200^{\circ} \mathrm{C}$ passou de $3,46 \times 10^{-16}$ m para $3,36 \times 10^{-15} \mathrm{~m}$ a $1650{ }^{\circ} \mathrm{C}$, significando um aumento de 10 vezes. A razão $\mathrm{k}_{2} / \mathrm{k}_{1}$ nas fatias internas da amostra passou, no mesmo intervalo de temperatura, de $7,23 \times 10^{3} \mathrm{~m}^{-1}$ para $2,51 \times 10^{4} \mathrm{~m}^{-1}$, correspondendo ao dobro da variação encontrada para $\mathrm{k}_{2} / \mathrm{k}_{1}$ nas fatias superficiais.

A explicação para a variação da permeabilidade entre $1200^{\circ} \mathrm{C}$ e $1650^{\circ} \mathrm{C}$ não está aqui relacionada à saída de água da estrutura, mas sim a dois fenômenos concorrentes que acontecem nesta faixa de temperatura. Por um lado, o processo de sinterização causa a modificação estrutural do concreto, fechando os poros deixados pela saída de água. No entanto, apesar de tanto a matriz quanto o agregado serem constituídos majoritariamente de um mesmo material (alumina), ambos apresentam sinterabilidade distinta devido à diferente granulometria e densidade de empacotamento. Esta diferença leva à geração de tensões residuais durante o resfriamento que culminam com o aumento da porosidade na interface matrizagregado [9]. O resultado final da competição entre sinterização e
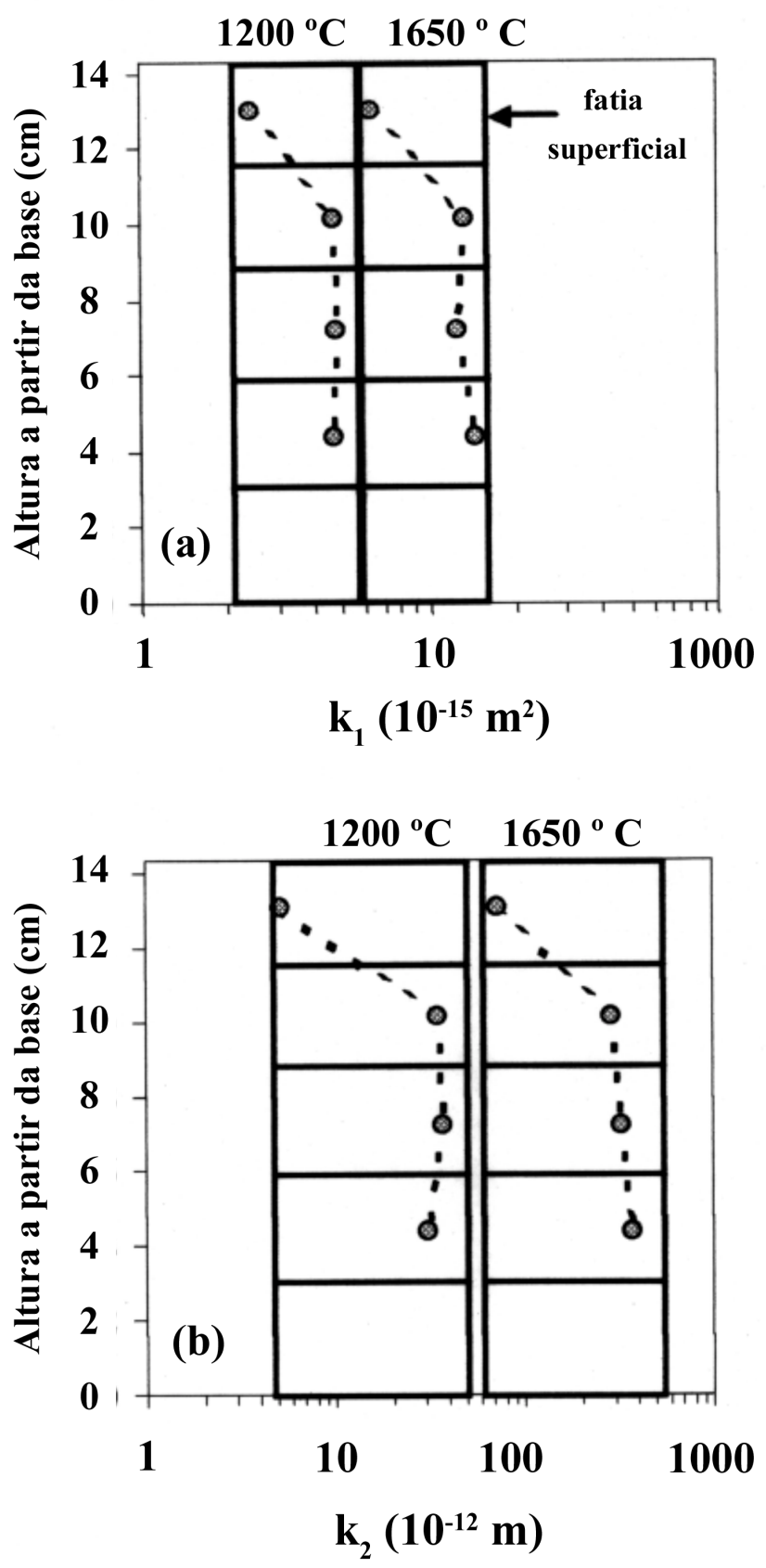

Figura 5: Variação das constantes de permeabilidade ao longo da espessura do concreto refratário tratado a $1200^{\circ} \mathrm{C}$ e $1650^{\circ} \mathrm{C}$. (a) Permeabilidade Darciana, $\mathrm{k}_{1}$. (b) Permeabilidade não-Darciana, $\mathrm{k}_{2}$.

[Figure 5: Permeability profile along the castable thickness for sample treated at $1200^{\circ} \mathrm{C}$ and $1650^{\circ} \mathrm{C}$. (a) Darcian permeability, $k_{1}$. (b) Non-Darcian permeability, $\left.k_{2}\right]$.

destacamento depende do mecanismo predominante na temperatura de queima. Pelos resultados encontrados neste trabalho, pode-se concluir que o mecanismo de destacamento matriz-agregado tenha sido dominante e responsável pelo aumento de permeabilidade observado entre $1200{ }^{\circ} \mathrm{C}$ e $1650{ }^{\circ} \mathrm{C}$.

A Fig. 6 evidencia as diferentes causas da variação de permeabilidade de um concreto refratário com o aumento de temperatura de tratamento térmico. Tendências similares são observadas para ambas as constantes, $\mathrm{k}_{1}$ (Fig. 6-a) e $\mathrm{k}_{2}$ (Fig. 6-b).

Entre $110^{\circ} \mathrm{C}$ e $900{ }^{\circ} \mathrm{C}$ ocorre a eliminação dos hidratos do concreto, tornando-o mais permeável pelos vazios deixados pela água liberada. A partir do início da sinterização, acima de $900{ }^{\circ} \mathrm{C}$, co- 

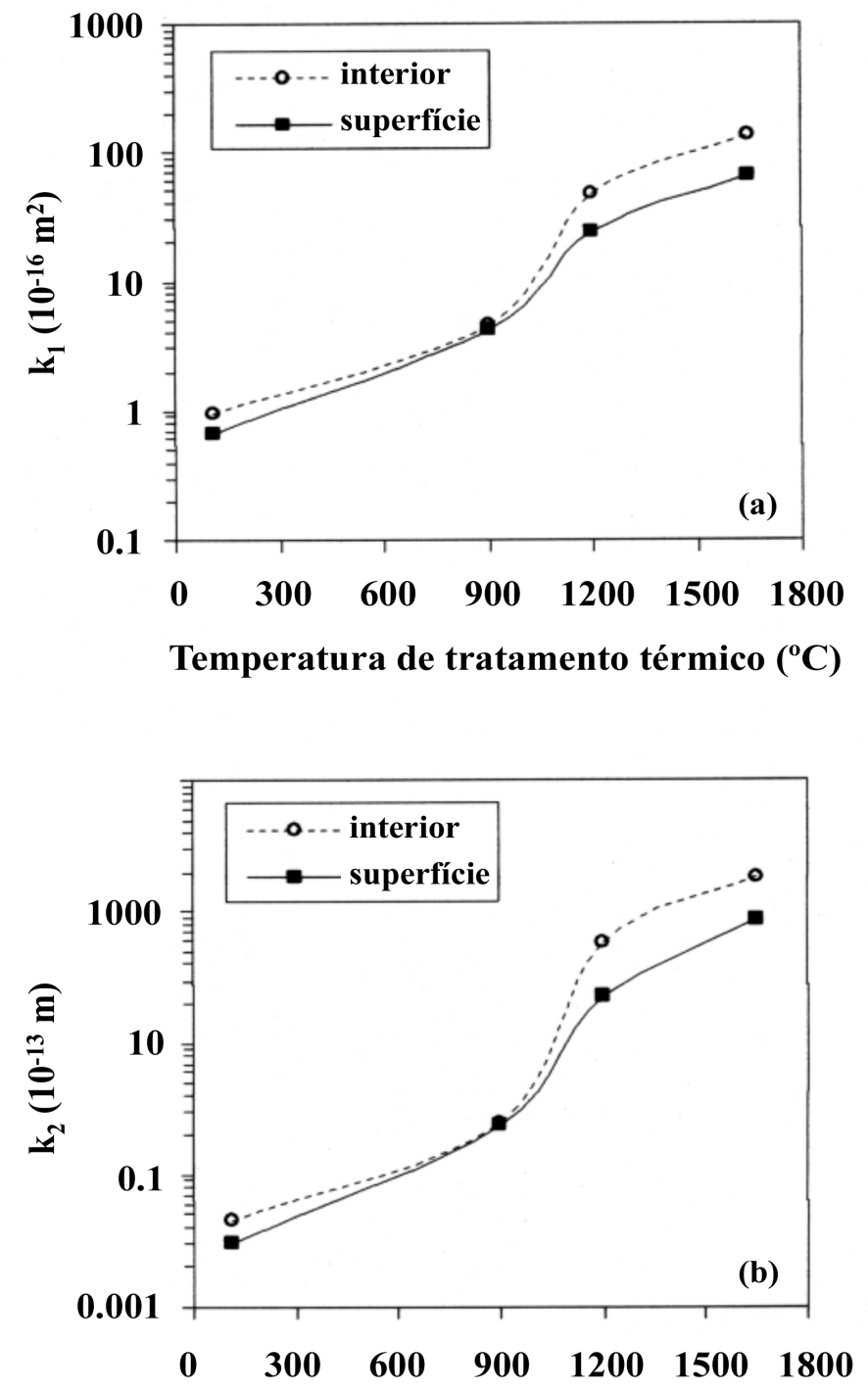

Temperatura de tratamento térmico $\left({ }^{\circ} \mathrm{C}\right)$

Figura 6: Evolução da permeabilidade no interior e na superfície das amostras analisadas neste trabalho como função da temperatura de tratamento térmico. (a) Permeabilidade Darciana, $\mathrm{k}_{1}$. (b) Permeabilidade não-Darciana, $\mathrm{k}_{2}$.

[Figure 6: Surface and inner permeability variation with the thermal treatment temperature. (a) Darcian permeability, $k_{1}$, (b) Non-Darcian permeability, $\left.k_{2}\right]$.

meça haver o fechamento dos poros pela reestruturação da matriz, que é compensada porém pela ocorrência do destacamento matrizagregado, resultando em novo aumento de permeabilidade. A predominância deste último mecanismo pode ser observada entre $1200^{\circ} \mathrm{C}$ e $1650^{\circ} \mathrm{C}$, quando há a inflexão das curvas de $\mathrm{k}_{1}$ e $\mathrm{k}_{2}$. Provavelmente, acima de $1650^{\circ} \mathrm{C}$ a geração de fases líquidas será dominante sobre o destacamento, permitindo que os poros sejam preenchidos e ocorra finalmente a diminuição de permeabilidade da estrutura [3].

A diferença entre a permeabilidade na superfície e no interior das amostras apresenta o mínimo na temperatura de $900{ }^{\circ} \mathrm{C}$, justamente por ser a região em que todos os vazios deixados pela água de hidratação na superfície do corpo ainda não sofreram o efeito da sinterização. Em temperaturas superiores a $900{ }^{\circ} \mathrm{C}$, a diferença de permeabilidade aumenta, pois, conforme discutido, a presença predominante de finos na camada superficial garante uma melhor sinterização. Já o interior da amostra, por possuir uma quantidade

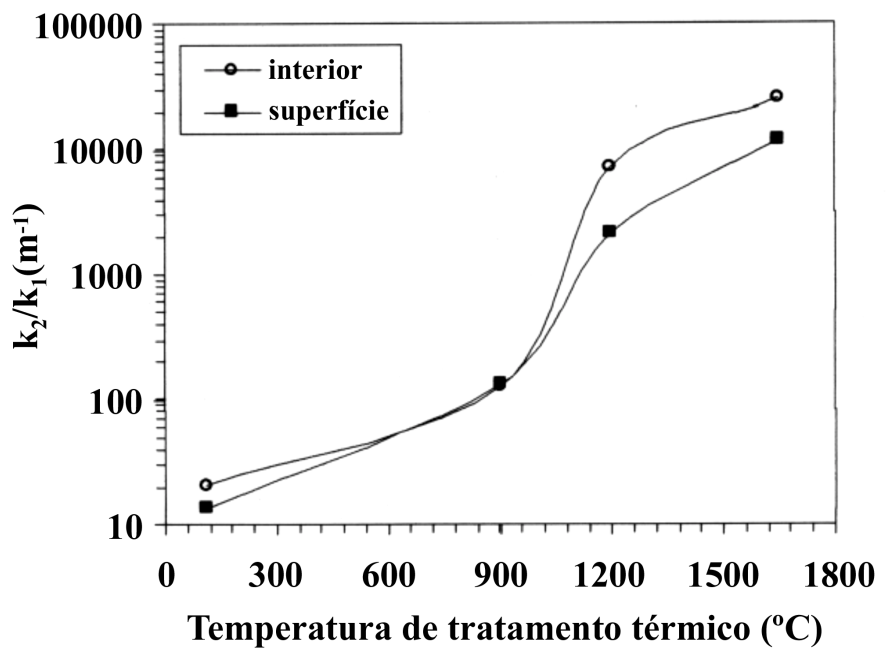

Figura 7: Variação da razão $\mathrm{k}_{2} / \mathrm{k}_{1}$ com a temperatura de tratamento térmico das amostras.

[Figure 7: Variation in the $k_{2} / k_{1}$ ratio with the sample thermal treatment temperature].

maior de agregados, sente mais o efeito do destacamento do mesmo com a matriz [3-9].

A Fig. 7 mostra a evolução da razão entre constantes de permeabilidade, $\mathrm{k}_{2} / \mathrm{k}_{1}$, como função da temperatura de tratamento térmico.

Considerando-se que essa razão de constantes indique qualitativamente a predominância de um determinado parâmetro da estrutura sobre a resistência ao escoamento do fluido, então, baseado na equação de Forchheimer, seria razoável afirmar que o trajeto percorrido pelo fluido torna-se menos tortuoso com a elevação da temperatura de queima da amostra. A conseqüência é que o termo parabólico, indicativo da ação inercial sobre a queda de pressão, passa a ter menos importância que o termo viscoso e linear para a mesma faixa de velocidades analisada. Embora na prática o escoamento de gases ou líquidos em concretos refratários seja lento, não é possível afirmar se ele é predominantemente viscoso e laminar antes que uma comparação com o termo inercial seja feita.

Embora não esteja ainda claro qual é a exata extensão da camada superficial de baixa permeabilidade, é inquestionável que sua presença afeta as propriedades de transporte em um concreto refratário. Em valores absolutos, pode-se considerar que entre $110^{\circ} \mathrm{C} \mathrm{e}$ $1650{ }^{\circ} \mathrm{C}$, quanto maior a temperatura de queima, maior foi a permeabilidade das amostras.

Em relação ao processo de secagem, a presença da camada superficial de baixa permeabilidade verificada neste trabalho pode indicar o acúmulo de tensões internas pelo aumento da pressão de vapor durante o aquecimento. No caso em que o tratamento térmico é realizado diretamente no local de aplicação do concreto, sem a existência de uma etapa prévia de secagem lenta e controlada, pode haver até mesmo a explosão do corpo refratário pelo retardamento da liberação de água para a superfície. A otimização das propriedades de transporte dependerá assim do compromisso entre eliminação de água durante a secagem e a infiltração de fluidos corrosivos durante a utilização do concreto refratário. A redução do perfil de permeabilidade é vista neste sentido como uma garantia de um meio poroso mais homogêneo e de maior previsibilidade em relação à percolação de fluidos. A adição de fibras (para aumentar a permeabilidade na superfície) ou a melhoria da adesão 
matriz-agregado (para diminuir a permeabilidade no interior) seriam alternativas para a minimização do perfil de permeabilidade e conseqüente otimização do concreto refratário.

\section{CONCLUSÕES}

Foi verificada neste trabalho a existência de perfil de permeabilidade ao longo da espessura de concretos refratários com ultrabaixo teor de cimento. As constantes de permeabilidade $\mathrm{k}_{1}$ e $\mathrm{k}_{2}$ da equação de Forchheimer tiveram aumento da superfície exposta à atmosfera para o interior, com intensidade dependente da temperatura de tratamento térmico do concreto. A eliminação de água de hidratação, a sinterização e o destacamento na interface matrizagregado foram os mecanismos dominantes para a elevação da permeabilidade entre $110^{\circ} \mathrm{C}$ e $1650{ }^{\circ} \mathrm{C}$. A leve sedimentação dos agregados para o interior das amostras e/ou o empacotamento diferenciado na camada superficial foram as causas prováveis para o gradiente de permeabilidade observado nas amostras estudadas.

\section{AGRADECIMENTOS}

Os autores agradecem a FAPESP, CNPq, CAPES e Alcoa Alumínio S.A. pelo apoio na realização deste trabalho.

\section{REFERÊNCIAS}

[1] R. E. Moore, J. D. Smith, W. L. Headrick Jr, T. P. Sandler, Proceedings of the 32nd Annual Symposium on Refractories, The American Ceramic Society, St. Louis Section, (1996) 26.

[2] M. D. M. Innocentini, V. C. Pandolfelli, Cerâmica 45, 292/293 (1999) 61-67.

[3] J. P. Singh, Advanced Ceramic Materials 3, 1 (1998) 18.

[4] O. Sudre, F. F. Lange, J. Am. Ceram. Soc. 80, 3 (1997) 800.

[5] R. E. Fisher, Am. Ceram. Soc. Bull. 59, 7 (1980) 702.

[6] M. D. M. Innocentini, A. R. F. Pardo, V. R. Salvini, V. C. Pandolfelli, Am. Ceram. Soc. Bull. 78, 11 (1999) 64.

[7] M. D. M. Innocentini, A. R. F. Pardo, V. C. Pandolfelli, Proceedings of the Unified International Technical Conference on Refractories UNITECR' 99, Berlin - Germany, September (1999) 93.

[8] M. D. M. Innocentini, A. R. F. Pardo, V. C. Pandolfelli, J. Am. Ceram. Soc. 83, 6 (2000) 1536.

[9] A. R. F. Pardo, M. D. M. Innocentini, V. C. Pandolfelli, Anais do $43^{\circ}$ Congresso Brasileiro de Cerâmica e $4^{\circ}$ Congresso de Cerâmica do Mercosul, Florianópolis - SC (1999) 12.

[10] M. D. M. Innocentini, A. R. Studart, R. G. Pileggi, V. C. Pandolfelli, $44^{\circ}$ Congresso Brasileiro de Cerâmica, Águas de São Pedro - SP (2000).

(Rec. 09/09/00, Ac. 10/11/00) 